Editorial

\title{
Special Issue on Computational Intelligence and Nature-Inspired Algorithms for Real-World Data Analytics and Pattern Recognition
}

\author{
Stefano Cagnoni ${ }^{1}$ (D) and Mauro Castelli ${ }^{2, *}$ (D) \\ 1 Dipartimento di Ingegneria e Architettura, Università degli Studi di Parma, 43124 Parma, Italy; \\ stefano.cagnoni@unipr.it \\ 2 NOVA Information Management School (NOVA IMS), Universidade Nova de Lisboa, \\ 1070-312 Lisboa, Portugal \\ * Correspondence: mcastelli@novaims.unl.pt
}

Received: 22 February 2018; Accepted: 24 February 2018; Published: 28 February 2018

\begin{abstract}
This special issue of Algorithms is devoted to the study of Computational Intelligence and Nature-Inspired Algorithms for Real-World Data Analytics and Pattern Recognition. The special issue considered both theoretical contributions able to advance the state-of-the-art in this field and practical applications that describe novel approaches for solving real-world problems.
\end{abstract}

Keywords: Computational Intelligence; nature-inspired algorithms; data analytics; pattern recognition

\section{Introduction}

Computational Intelligence (CI) [1] and Nature-Inspired Computation (NIC) [2] are mature branches of Artificial Intelligence. The main feature common to the techniques they deal with is that, given a problem, they mimic a natural system or process to construct a solution that is optimal for both quality and robustness. The analogies and abstractions developed in these fields have been able to provide valuable insights for successful algorithmic design and improvement, in many cases outperforming traditional search and heuristics. Relevant examples include fuzzy systems [3], evolutionary algorithms [4], and neural networks [5]. State-of-the-art developments in nature-inspired algorithms and their applications in various disciplines are described in recent literature [6].

CI and NIC have been demonstrated to be able to produce human-competitive results [7], as has happened with neural models that have led to the development of Deep Learning [8], or with the study of artificial evolution and the development of Genetic Algorithms [9] and Genetic Programing [10]. These techniques have been particularly successful in the fields of Computer Vision and Pattern Recognition [11-13] and Data Analytics [14-16].

\section{Special Issue}

The aim of this Special Issue is to gather and present recent work where CI and NIC algorithms are specifically designed for, or applied to, solving complex real-world problems in Data Analytics and Pattern Recognition, by means of: (1) state-of-the-art methods having general applicability; (2) domain-specific solutions; or (3) hybrid algorithms that integrate CI and NIC with traditional numerical and mathematical methods.

In response to the call for papers, we selected six submissions for the special issue, all of which are of high quality, reflecting the growing interest in the area of CI and NIC for real-world data analytics and pattern recognition. All submissions were reviewed by at least three experts.

The first paper investigates the feasibility of classifying (inferring) the emergency braking situations in road vehicles from the motion pattern of the accelerator pedal; the second paper proposes 
a system based on an Deep Extreme Learning Machine Network for fabric weave pattern and yarn color recognition and classification; the third paper addresses the variable selection problem in time series forecasting using random forests; the fourth paper introduces a game theory-inspired evolutionary algorithm for global optimization; the fifth paper presents a genetic algorithm-based technique for estimating the two-view epipolar-geometry of uncalibrated perspective stereo images from putative correspondences containing a high percentage of outliers; and the last paper introduces an improved brain-inspired emotional learning algorithm for fast classification.

Acknowledgments: As Guest Editors, we would like to thank the authors for submitting their work to the special issue, the reviewers for their constructive comments, and the editorial staff of MDPI Algorithms Journal for their assistance in managing the review process and for their continuous support whenever needed.

Conflicts of Interest: The authors declare no conflict of interest.

\section{References}

1. Fulcher, J. Computational intelligence: An introduction. In Computational Intelligence: A Compendium; Springer: Berlin/Heidelberg, Germany, 2008.

2. Yang, X.S. Nature-Inspired Metaheuristic Algorithms; Luniver Press: Frome, UK, 2010.

3. Nauck, D.; Klawonn, F.; Kruse, R. Foundations of Neuro-Fuzzy Systems; John Wiley \& Sons, Inc.: Hoboken, NJ, USA, 1997.

4. Back, T. Evolutionary Algorithms in Theory and Practice: Evolution Strategies, Evolutionary Programming, Genetic Algorithms; Oxford University Press: Oxford, UK, 1996.

5. Bishop, C.M. Neural Networks for Pattern Recognition; Oxford University Press: Oxford, UK, 1995.

6. Yang, X.S. Nature-Inspired Algorithms and Applied Optimization; Springer: Berlin/Heidelberg, Germany, 2017.

7. Koza, J.R.; Keane, M.A.; Streeter, M.J.; Mydlowec, W.; Yu, J.; Lanza, G. Genetic Programming IV: Routine Human-Competitive Machine Intelligence; Springer Science \& Business Media: Berlin, Germany, 2006.

8. LeCun, Y.; Bengio, Y.; Hinton, G. Deep learning. Nature 2015, 521, 436. [CrossRef] [PubMed]

9. Goldberg, D.E.; Holland, J.H. Genetic algorithms and machine learning. Mach. Learn. 1988, 3, 95-99. [CrossRef]

10. Koza, J.R. Genetic programming as a means for programming computers by natural selection. Stat. Comput. 1994, 4, 87-112. [CrossRef]

11. Cagnoni, S. Evolutionary computer vision: A taxonomic tutorial. In Proceedings of the Eighth International Conference on Hybrid Intelligent Systems, HIS 2008, Barcelona, Spain, 10-12 September 2008.

12. Cagnoni, S.; Dobrzeniecki, A.B.; Poli, R.; Yanch, J.C. Genetic algorithm-based interactive segmentation of 3D medical images. Image Vis. Comput. 1999, 17, 881-895. [CrossRef]

13. Cagnoni, S.; Mordonini, M.; Sartori, J. Particle swarm optimization for object detection and segmentation. In Workshops on Applications of Evolutionary Computation; Springer: Berlin/Heidelberg, Germany, 2007.

14. Castelli, M.; Vanneschi, L.; De Felice, M. Forecasting short-term electricity consumption using a semantics-based genetic programming framework: The South Italy case. Energy Econ. 2015, 47, 37-41. [CrossRef]

15. Castelli, M.; Castaldi, D.; Giordani, I.; Silva, S.; Vanneschi, L.; Archetti, F.; Maccagnola, D. An efficient implementation of geometric semantic genetic programming for anticoagulation level prediction in pharmacogenetics. In Portuguese Conference on Artificial Intelligence; Springer: Berlin/Heidelberg, Germany, 2013.

16. Castelli, M.; Vanneschi, L. Genetic algorithm with variable neighborhood search for the optimal allocation of goods in shop shelves. Oper. Res. Lett. 2014, 42, 355-360. [CrossRef] 